\title{
The electromagnetic response of resonance matter and other strange observations
}

\author{
Tetyana Galatyuk* \\ Technische Universität Darmstadt, Germany \\ GSI Helmholtzzentrum für Schwerionenforschung GmbH, Germany \\ E-mail: tgalatyukeikp.tu-darmstadt.de
}

\section{for the HADES collaboration ${ }^{\dagger}$}

The matter formed in central heavy-ion collisions at a few $\mathrm{GeV}$ per nucleon is commonly understood as resonance matter, a gas of nucleons and excited baryonic states with a substantial contribution from mesonic, mostly pionic excitations. Yet, in the initial phase of the reaction the system is compressed to beyond nuclear ground state density and hence substantial modifications of the hadron properties are expected to occur. It is conjectured that at high enough densities hadronic degrees of freedom would finally disappear and a chirally restored phase of quarks would emerge. In this contribution we present key results on in-medium properties of hadrons obtained by the High Acceptance DiElectron Spectrometer. The spectral distribution of virtual photons emitted from the collision zone of $\mathrm{A}+\mathrm{A}$ collisions indicates strong medium effects beyond those resulting from a pure superposition of individual $\mathrm{N}+\mathrm{N}$ collisions. This observable, as well as the measured hadron abundances in the final state, show features of a thermalized fireball. Baryon-driven medium effects influence significantly the $\rho$ meson in-medium spectral function and are considered essential in describing the low-mass dilepton spectra. While the measured abundance of all reconstructed particles are well described assuming thermalization, the double strange cascade $\Xi^{-}$(1321) production in $\mathrm{A}+\mathrm{A}$ and $\mathrm{p}+\mathrm{A}$ collisions exhibits a sizeable enhancement above predictions of statistical hadronization and transport model calculations. A deeper understanding of the microscopic properties of resonance matter requires systematic investigation of baryonic decays and these are studied in HADES making use of pion beams. This experimental program will be continued in the coming years with the upgraded HADES detector.

The 26th International Nuclear Physics Conference

11-16 September, 2016

Adelaide, Australia

\footnotetext{
* Speaker.

${ }^{\dagger}$ The collaboration gratefully acknowledges the following funding: INFN-LNS Catania (Italy); LIP Coimbra (Portugal): PTDC/FIS/113339/2009; SIP JUC Cracow (Poland): 2013/10/M/ST2/00042; GSI Darmstadt (Germany): Helmholtz Alliance HA216/EMMI; TU Darmstadt (Germany): VH-NG-823, Helmholtz Alliance HA216/EMMI; HZDR, Dresden (Germany): 283286, 05P12CRGHE; Goethe-University, Frankfurt (Germany): Helmholtz Alliance HA216/EMMI, HIC for FAIR (LOEWE), GSI F\&E, BMBF 06FY9100I; TU München, Garching (Germany), MLL München: DFG EClust 153, DFG FAB898/2-1, BmBF 05P15WOFCA; JLU Giessen (Germany): BMBF:05P12RGGHM; University Cyprus, Nicosia (Cyprus): UCY/3411-23100; IPN Orsay, Orsay Cedex (France): CNRS/IN2P3; NPI AS CR, Rez, (Czech Republic): GACR 13-06759S, MSMT LM2015049.
} 


\section{Searching for landmarks of the QCD phase diagram of matter}

It is a great challenge to understand the nature of processes which led to the creation of the world around us. Research on nuclear and quark matter at high net-baryon density is expected to put our empirical understanding of the origin of matter in the Universe to a more fundamental level. Consequently, during the last decades, substantial theoretical and experimental efforts have been devoted to the study of nuclear matter far from its ground state. A quantitative picture of the phase diagram of strongly interacting matter as a function of temperature and baryochemical potential $\mu_{B}$ (which is a measure for the net-baryon density) is shown in Figure 1. Matter was formed in the early Universe about ten microseconds after the Big Bang with vanishing baryochemical potential and thereafter traversed the phase diagram essentially downwards along the temperature axis. On the other hand, compact stellar objects like neutron stars, are comparatively cold and contain forms of matter in their interior located in the region of large baryochemical potential.

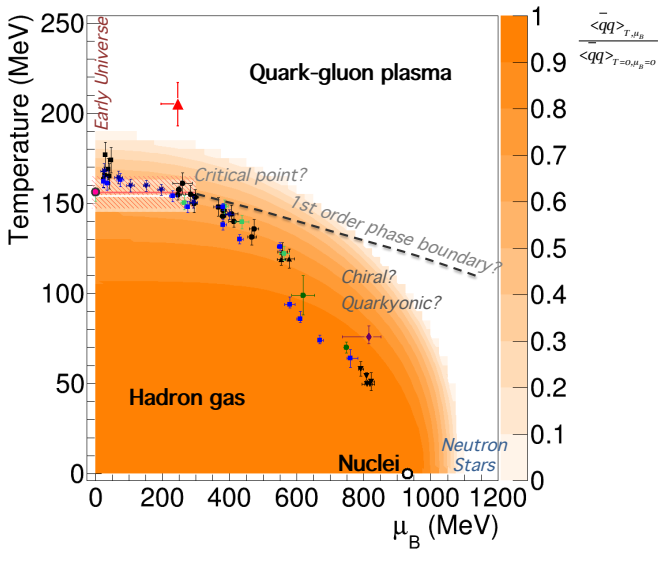

Figure 1: Data points in $\mathrm{T}$ and $\mu_{B}$ describing the final state hadron abundances in a statistical hadronization model (blue squares and pink circle from [1], light green squares from [2], black solid symbols from [3], dark blue stars from [4], green circles [5], lila diamond [6], Lattice QCD results from [7] (white curve) and [8] (red curve)). The red triangle is the point measured from the invariant-mass slope of dimuons in the mass range $1.2<\mathrm{M}_{\mu \mu}<2 \mathrm{GeV} / \mathrm{c}^{2}$ measured in In+In collisions at $\sqrt{s_{N N}}=17.2 \mathrm{GeV}$ [9]. The expectation value of the chiral condensate relative to the vacuum is depicted as orange gradient [11].

At moderate temperatures and baryochemical potentials, Quantum Chromodynamics (QCD) is a strongly coupled theory, quarks and gluons are confined into hadrons, and the chiral symmetry of QCD is spontaneously broken by the non-vanishing quark condensate (see Figure 1, orange gradient is equal to 1 in this region). At high $\mathrm{T}$ and/or $\mu_{B}$, the coupling constant becomes small, quarks and gluons are liberated, and chiral symmetry is restored. This result in a new state of matter, the quark-gluon plasma (QGP). For baryochemical potential $\mu_{B}=0$, first principle Lattice-QCD calculations predict that the restoration of chiral symmetry happens via a crossover transition at the temperature of $\mathrm{T}=155 \pm 9 \mathrm{MeV}$. At small $\mathrm{T}$ and $\mu_{B}$ of order $1 \mathrm{GeV}$ the calculations from QCDinspired effective field theories predict that the chiral transition is of first order. The first-order phase boundary ends in a second-order critical point [10], the location of which is presently not accessible to first-principle QCD calculations and varies from model to model. At low temperatures and baryochemical potentials exceeding that of the nuclear matter ground state, a new phase, named "quarkyonic phase" was recently proposed $[12,13,14,15]$. The concept of such chirally symmetric but still confined phase is currently subject to theoretical debate and remains to be addressed experimentally. Moreover, in [16] it has been suggested that at collision energies of several $\mathrm{GeV}$ per nucleon the bulk of the system will reside within the spinodal region of the phase diagram. Furthermore, as discussed in [17], the presence of an inhomogeneous island at intermediate chemical potentials and low temperatures could occur. 
Collisions of heavy-ions at relativistic energies are the only possibility, at least on the Earth, to recreate extreme states of matter and allow us to study phenomena relevant also to other areas of physics, like cosmology and astrophysics, under controlled laboratory conditions. Heavy-ion experiments at accelerator facilities like RHIC at BNL, SPS and LHC at CERN and SIS18 at GSI are currently carried out. The interest to explore the first-order phase transition region and to find the location of critical end point has recently dramatically increased. New generation accelerators are planned or being constructed in Germany (FAIR), Japan (J-PARC) and Russia (NICA).

At bombarding energies of $\mathrm{E}_{l a b} \simeq 1-2 A \mathrm{GeV}$ (the current SIS18) a large region in the phase diagram of matter, ranging from ground state matter density $\rho_{0}$ up to about $3 \rho_{0}$, can be accessed with a proper choice of the collision system. Here, the matter in the central reaction volume reaches temperatures $\mathrm{T} \leq 80 \mathrm{MeV}$, very likely without reaching the QGP phase boundary. On the other hand, a substantial depletion of the chiral condensate is conjectured already at SIS18 energies, making this region highly interesting. Remarkably, the state of QCD matter at such conditions resembles the one of neutron star mergers [18]. Therefore heavy-ion program at SIS18 energies complements studies of the nuclear matter equation of state (EoS) in extreme environments.

\section{The HADES strategy}

The challenge remains to detect various phases in the laboratory by isolating their unambiguous signals. Among the promising observables for investigating the microscopic properties of strongly interacting matter are virtual photons materializing by formation of lepton pairs inside the hot and dense matter (for review see [19]). Hadrons carrying strangeness are valuable messengers as well, because at incident energies of few $\mathrm{GeV}$ per nucleon their production threshold is mostly above the energy available in a single (free) $\mathrm{N}+\mathrm{N}$ collision and therefore (i) requires a certain degree of collectivity in the system, (ii) depends on the nuclear matter EoS, in-medium potentials, and hadron in-medium properties [20, 21].
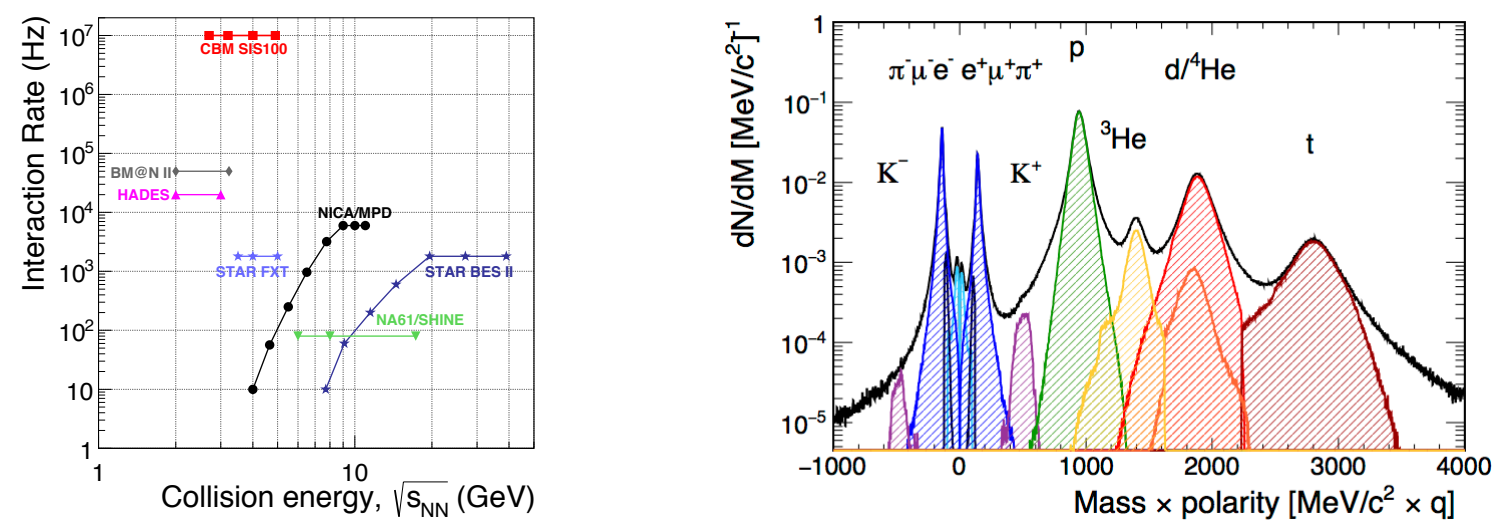

Figure 2: Left: Interaction rates achieved by existing and planned heavy-ion experiments exploring high $\mu_{B}$ region as a function of center-of-mass energy [23]. High-rate experiments are also proposed at J-PARC [24] and at SPS [25], but are still in a conceptual stage. Right: Mass times polarity of the reconstructed particles in $\mathrm{Au}+\mathrm{Au}$ collisions at $1.23 \mathrm{~A} \mathrm{GeV}$. 
The High Acceptance DiElecton Spectrometer (HADES), installed at heavy-ion synchrotron SIS18 at the GSI Helmholtzzentrum für Schwerionenforschung (Germany), is currently the only experiment studying properties of strongly interacting matter in a few $A \mathrm{GeV}$ energy regime. It is a unique apparatus to study dielectron $\left(e^{+} e^{-}\right)$and hadron production in heavy-ion collisions, as well as in proton- and pion-induced reactions in the energy range of $1-4 \mathrm{GeV}$. HADES is a fixed-target experiment with large angular acceptance $\left(18^{\circ}-80^{\circ}\right.$ in polar and almost full in azimuthal angles). The rate capabilities of HADES are presented in Fig. 2 (left panel). The setup comprises a Ring Imaging Cherenkov Detector (RICH) in the field-free region around the target, a low-mass tracking system, a superconducting toroid, and additional detectors for particle identification (for details see [22]). In Fig. 2 (right panel) we present the mass $\times$ polarity distribution of the reconstructed particles. The data quality regarding statistics and mass resolution (e.g. of $\left.\sigma\left(\mathrm{M}_{\text {pole }}^{\omega}\right)=16 \mathrm{MeV} / c^{2}\right)$ provides high sensitivity to the (in-medium) spectral function of vector mesons.

In order to understand the microscopic structure of matter in the region of high baryochemical potential HADES pursues a strategy, which relies on systematic measurements of strangeness production and virtual photon emission in elementary and heavy-ion collisions. As of now, HADES has measured rare and penetrating probes in $\mathrm{p}+\mathrm{p}, \mathrm{n}+\mathrm{p}, \mathrm{C}+\mathrm{C}, \mathrm{p}+\mathrm{Nb}, \mathrm{Ar}+\mathrm{KCl}$ and $\mathrm{Au}+\mathrm{Au}$ collisions.

\section{Electromagnetic radiation}

Electromagnetic probes (photons and dileptons) are a powerful tool to identify and characterize microscopic properties of strongly interacting matter. Short-lived vector mesons ( $\rho, \omega$ and $\phi)$ couple directly to the electromagnetic current. Unlike hadrons, dileptons do not interact strongly leading to large mean free paths much larger than the spatial extent of the fireball created in a heavyion collision. Once vector mesons have decayed into a purely leptonic final state, the daughter particles traverse the dense medium without substantial final state interaction. Hence, dileptons carry information about the entire space-time history of the collision. By means of their 4-momentum reconstructed from the lepton pair, such states provide a direct link to the properties of the medium. Baryon-driven medium effects influence significantly the $\rho$ meson in-medium spectral function and are considered as the key to describing the low-mass dilepton spectra measured at SPS energies [26]. An extension of dilepton spectroscopy to higher collision energies was achieved at RHIC [27, 28]. The recent STAR data from the RHIC beam energy scan constitute a first excitation function, establishing the consistency with previous SPS results [29]. The successful $\rho$ mass broadening scenario implies a strong coupling of the $\rho$ meson to baryons [30, 31, 32, 33, 34]. Quantitative descriptions of heavy-ion collisions at bombarding energies of a few $\mathrm{GeV}$ per nucleon indicate that, due to the nucleon stopping, the dominant medium radiation is presumably driven by baryonic excitations (resonances) in the system and therefore baryon-driven medium effects are expected to be maximal.

\subsection{HADES results from $\mathrm{N}+\mathrm{N}$ collisions}

Measurements in $\mathrm{N}+\mathrm{N}$ collisions at various beam energies provided valuable constraints on sources contributing to the dielectron signal and allowed to obtain model-independent reference spectra for studies of "cold" nuclear matter ( $\mathrm{p}+\mathrm{A}$ collisions) and hot and dense matter effects ( $\mathrm{A}+\mathrm{A}$ collisions). To better understand the contributions to dielectron yield from first chance collisions 

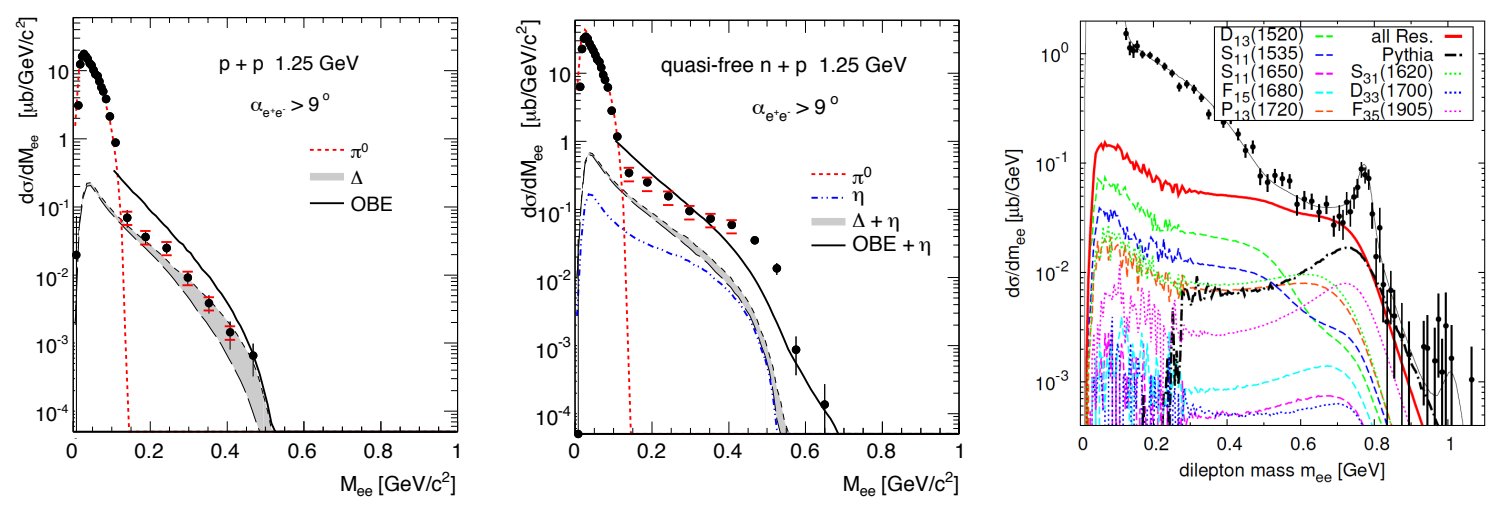

Figure 3: Left: Electron pair differential cross sections as function of invariant mass (full circles) measured in $\mathrm{p}+\mathrm{p}$ reactions at $1.25 \mathrm{GeV}$ [50]. Middle: Same in quasi-free $\mathrm{n}+\mathrm{p}$ reactions at $1.25 \mathrm{GeV}$ [50]. Two assumptions for the $\Delta$ nucleon transition form factor (magnetic dipole: long dashed, Vector Dominance Model: dashed), and a full OBE model calculation (full line). The $\eta$ Dalitz contribution is added to the model calculations and also shown separately (dashed-double dotted). Right: Same in p+p collisions at $3.5 \mathrm{GeV}$ [35] compared to a calculation using a resonance model as implemented in GiBUU microscopic transport code [36]. All baryonic resonances, except the $\Delta(1232)$, produce lepton pairs via explicit decay to an intermediate meson and subsequent annihilation into a pair of electrons.

(i.e. the early stage of heavy-ion collisions), HADES studied $\mathrm{p}+\mathrm{p}$ and quasi-free $\mathrm{n}+\mathrm{p}$ interactions at $E_{k i n}=1.25 \mathrm{GeV}$, an energy chosen such that $\eta$ meson production is sub-threshold in protonproton reactions [50]. The main goal of the latter experiment is to understand the conventional $\mathrm{n}+\mathrm{p}$ bremsstrahlung component for $e^{+} e^{-}$production in the tagged reaction channel $n+p \rightarrow n p$ $e^{+} e^{-}$and to establish an experimental cocktail of dielectrons from "free", i.e. non-medium hadron decays for SIS energies. Indeed, HADES has observed a surprisingly large isospin dependence comparing $\mathrm{p}+\mathrm{p}$ (Fig. 3.1, left panel) with $\mathrm{n}+\mathrm{p}$ (Fig. 3.1, middle panel) collisions. While conventional bremsstrahlung [37, 38] cannot account for the effect, calculations including emission from the internal charged pion line in an one-boson-exchange model for the $\mathrm{n}+\mathrm{p}$ case [39], and using a Vector Dominance Model (VDM) form factor for the pion-photon vertex, could reproduce the trend observed in the data. In a strict VDM picture such a process can be interpreted as the formation of a deep off-shell $\rho$ meson. This contribution is possible because, in contrast to $\mathrm{p}+\mathrm{p}$ reaction an exchange of charged pion is allowed.

Towards higher incident proton energy, i.e. $3.5 \mathrm{GeV}$, the situation changes substantially. Not only $\Delta$, but several higher lying baryonic resonances are active in $e^{+} e^{-}$-pair production. Figure 3.1 (right panel) shows the electron pair differential cross section obtained by HADES in the $p+p \rightarrow$ $e^{+} e^{-} X$ channel as a function of the dilepton invariant mass. The microscopic transport model calculations shown here represent prompt dileptons that stem solely from (off-shell) $\rho$ meson production produced in baryonic resonance decays obtained from the two-component model [40]. The treatment of the off-shell $\rho$ meson propagation leads to the strongly modified mass distribution. Please note, that the model fails to describe the data if only the non-resonant production is assumed (PHYTIA, Figure 3.1 (right panel) dotted-dashed curve). However, this result has to be taken with caution since the relevant production cross sections of the resonances and their decay branches into 
$\rho N$ are to a large extent unknown and new precise data on elementary production amplitudes are urgently needed. It is expected that the recently collected data on dielectron [41] and pion production [42] in $\pi^{-}+p$ reaction channels and the planned future campaigns with the pion beam will shed more light on this important aspect.

\subsection{Are there narrow in-medium vector meson states with substantially shifted pole mass?}

A strong momentum dependence of the $\rho$ meson spectral function already at normal nuclear matter density $\rho=\rho_{0}$ has been predicted [43,44]. Data on the $\rho$ meson has been obtained in two experiments at KEK [45] and JLab [46]. These results are, however, controversial. One of the objections is that some of detector systems have no or little acceptance for low meson momenta for which the strongest medium modifications are expected. For the first time dielectons in the relevant momentum range $p_{e e}<0.8 \mathrm{GeV} / c$ have been measured by HADES in $\mathrm{p}+\mathrm{Nb}$ reactions at a kinetic beam energy of $3.5 \mathrm{GeV}$ [47]. The $\mathrm{p}+\mathrm{p}$ reactions at the same beam energy have been measured and serve as a necessary reference for the study in-medium modifications of vector mesons [35, 48]. Fig. 3.3 (left panel) shows an excess yield of electron pairs with momenta $p_{e e}<0.8 \mathrm{GeV} / c$ below the omega pole mass when comparing the spectral distribution of the $e^{+} e^{-}$invariant masses reconstructed in $\mathrm{p}+\mathrm{Nb}$ collisions to pairs produced in elementary $\mathrm{p}+\mathrm{p}$ reactions. Complementary to the results obtained by the NA60 collaboration [49], HADES sees rather melting than shift of the $\rho$ meson. These trends hint at strong coupling of the $\rho$ meson to baryonic resonances and absorption of the $\omega$ meson, both being manifestations of in-medium modifications of vector mesons.

\subsection{Virtual photon emission in A+A collisions}

An important prerequisite to identify true in-medium radiation of dense matter is the detailed knowledge of the complex superposition of baryonic and long-living mesonic contributions to the rate at various stages of the collision. In this context, we compare $e^{+} e^{-}$production rates measured in $\mathrm{C}+\mathrm{C}$ and $\mathrm{Ar}+\mathrm{KCl}$ collisions with the properly scaled production rates from $\mathrm{N}+\mathrm{N}$ collisions. To do so, the expected contributions from the $\eta$-Dalitz decay have been subtracted and a reference spectrum has been generated by computing the isospin-averaged emission probability per produced neutral pion from separately measured $p+p$ and quasi-free $n+p$ collisions. This reference spectrum and the yield observed in $\mathrm{C}+\mathrm{C}$ collisions coincide within the systematic uncertainty of about $25 \%$ [50]. This suggests that radiation components beyond that of binary nucleon collisions is not yet observed in this light collision system. Furthermore, this result, which provides a solution to the long standing "DLS puzzle", has its origin in a strong isospin dependence of $\mathrm{N}+\mathrm{N}$ bremsstrahlung, which is a particular feature of this energy regime [37, 39]. In contrast, a direct comparison of the $\mathrm{N}+\mathrm{N}$ reference spectrum with the invariant-mass distribution measured in $\mathrm{Ar}+\mathrm{KCl}$ collisions at $1.76 \mathrm{~A} \mathrm{GeV}$ shows a pronounced radiation excess between the $\pi^{0}$ Dalitz and the vector meson regions [51] (see Fig. 3.3, middle panel). In order to better quantify this excess radiation, we first subtract the cocktail of contributions from long-lived sources that has been obtained in the following way: (i) $\pi^{0}$ and $\eta$ meson multiplicities have been taken from a measurement discussed in $[52,53]$; (ii) the $\omega$ yield has been fixed such as to get, after subtraction, a smooth underlying continuum; the $\rho$ contribution is not subtracted. The dilepton excess radiation resulting from this analysis procedure is presented in Fig. 3.3 (right panel) and is characterized by nearly exponential shape. 

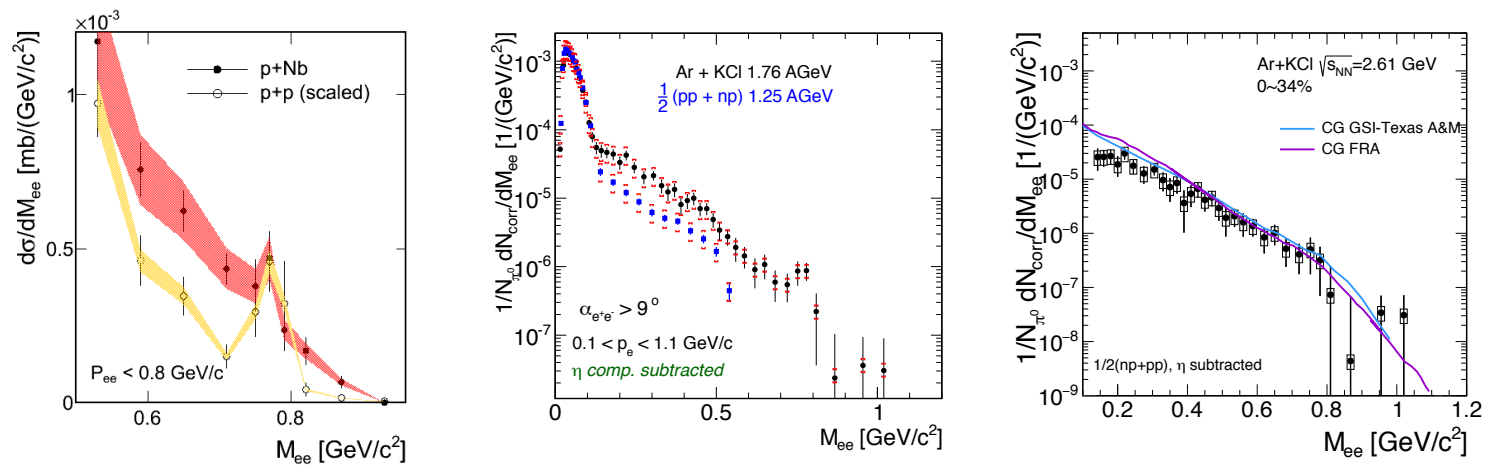

Figure 4: Left: Comparison of the invariant mass distribution of $e^{+} e^{-}-$pairs with $p_{e e}<0.8 \mathrm{GeV} / c$ in $\mathrm{p}+\mathrm{p}$ (open circles) and $\mathrm{p}+\mathrm{Nb}$ (full circles) collisions at the same kinetic beam energy of $3.5 \mathrm{GeV}$. Middle: Comparison of the reference spectrum from properly scaled $\mathrm{N}+\mathrm{N}$ collisions with the reconstructed $e^{+} e^{-}$ mass distribution in $\mathrm{Ar}+\mathrm{KCl}$ (the expected contribution from the $\eta$-Dalitz decay has been subtracted). Right: Dilepton excess radiation observed in the $34 \%$ most central $\mathrm{Ar}+\mathrm{KCl}$ collisions. Contributions from first chance $\mathrm{N}+\mathrm{N}$ collisions and late $\eta$-Dalitz decays have been subtracted from the data. The yield is normalized to the number of produced $\pi^{0}$ (to remove trivial $<\mathrm{A}_{\text {part }}>$ dependences). The blue [54] and pink [55] curves show the results of two versions of coarse grained UrQMD calculations using different concepts for obtaining the thermal parameters.

The excess radiation can be interpreted by assuming emission from a thermalized medium. The spectra observed in the experiment result then from a four-volume integral over the emissivity, weighted with the time-dependent temperature and density profile of the hot and dense system. The latter can e.g. be derived by coarse-grained microscopic transport calculations [54, 55]. The approach agrees well with data. Under this assumption, the emissivity of matter also at not too high temperatures is given by the thermal average of the in-medium propagator for which $\rho$-baryon couplings are fundamentally important. As a matter of fact, it is the penetrating nature of the dileptons, what allows to observe an effect of "shining" of the baryon-rich matter integrated over the whole collision time. As shown by the model calculations $[26,54]$ the yield of radiation is a direct measure of life-time of hot and dense fireball and therefore serves as a chronometer of the reaction. An important test of this scenario will be provided by data recently obtained from $\mathrm{Au}+\mathrm{Au}$ collisions at $1.25 \mathrm{~A} \mathrm{GeV}$ where even stronger contribution of thermal radiation is expected. High statistics will allow to characterize the dielectron yield beyond its mass distribution and to compare the data to predictions from various model calculations [56, 57].

\section{Hadron chemistry}

Baryonic resonances play an important role for the description of strangeness production in heavy-ion collisions, in particular at beam energies where the total energy in a single $\mathrm{N}+\mathrm{N}$ collision is not sufficient to produce a final state with strangeness [20]. For the $\mathrm{p}+\mathrm{Nb}$ and $\mathrm{Ar}+\mathrm{KCl}$ collision systems, most complete (at few $\mathrm{GeV}$ energy regime) sets of hadron yields containing strangeness have been reconstructed by HADES and is shown in Fig. 4 (left and middle panels). A surprisingly strong $\phi$ meson production has been extracted from an analysis of the $\mathrm{K}^{+} \mathrm{K}^{-}$final state. The 

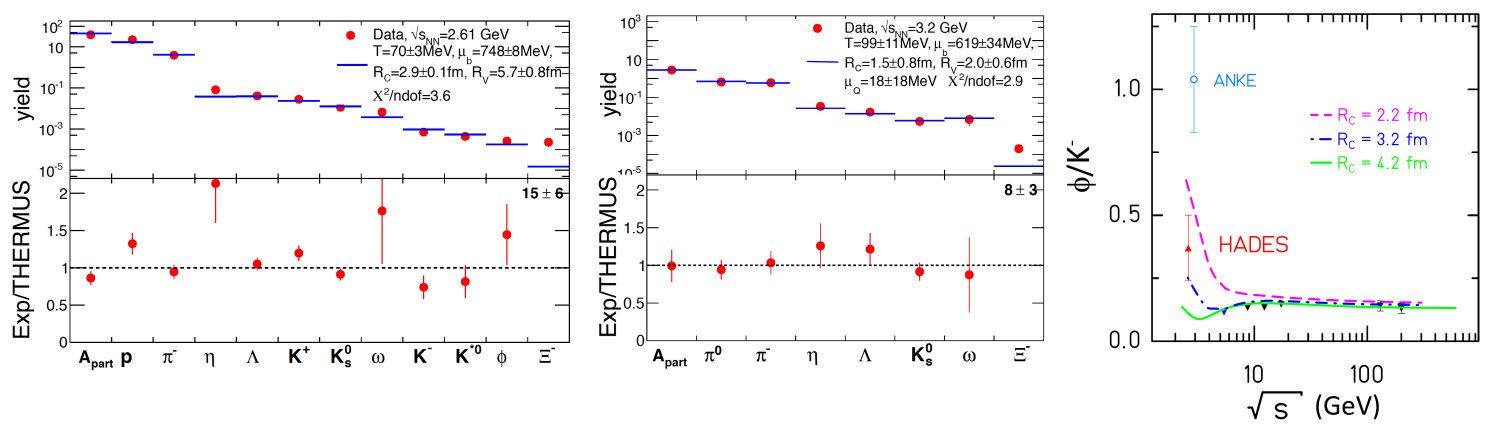

Figure 5: Left: Yields (full red circles) of hadrons produced in $\mathrm{Ar}+\mathrm{KCl}$ collisions at $1.76 \mathrm{~A} \mathrm{GeV}$ and the corresponding values of THERMUS fit (blue horisontal bars). The lower panel shows the ratio of the experimental values to the THERMUS values. Middle: Same in $\mathrm{p}+\mathrm{Nb}$ collisions at $3.5 \mathrm{GeV}$. Right: $\phi / \mathrm{K}^{-}$ ratio as a function of center-of-mass energy. The full red triangle shows the HADES point. The curves are predictions of an SHM for three values of the correlation radius parameter $R_{c}$.

acceptance corrected $\phi / \mathrm{K}^{-}$ratio has been found to be $0.37 \pm 0.13$ (see Fig. 4, right panel) which translates to fraction of $18 \pm 7 \%$ of negative kaons originating from $\phi$ decays. Furthermore, the observation of the different slopes of transverse mass distributions of kaon and anti-kaon spectra can naturally be explained as the effect of the $\phi$ feed-down [58]. This stays in opposition to the previously commonly accepted assumption, that the strangeness exchange process is the dominant mechanism of the $\mathrm{K}^{-}$production. HADES result reveals that $\phi$ meson production requires a multiparticle processes. This conclusion is supported by rather complex microscopic transport model calculations $[64,65]$.

On the other hand, the multiplicities of all observed hadrons (including $\phi$ ) can be explained assuming a break-up (freeze-out) of a thermalized hadronic system if strangeness is treated canonically by implementing a strangeness correlation radius $R_{c}$. The latter leads to suppression of hadronic states with open strangeness but not of states with hidden strangeness like the $\phi$ meson. The hadron abundances in $\mathrm{Ar}+\mathrm{KCl}$ collisions at $1.76 \mathrm{~A} \mathrm{GeV}$, but also in $\mathrm{p}+\mathrm{Nb}$ collisions at $3.5 \mathrm{GeV}$, are in a good agreement with the statistical hadronization model (SHM) [59] as shown in Fig. 4 (left and middle panels). The extracted temperatures and baryochemical potentials (Fig. 1 green circles) are found to be in agreement with the universal freeze-out curve. Since strangeness equilibration requires a long time interval (up to $20-40 \mathrm{fm} / \mathrm{c}$ [60]), an understanding of the apparent equilibration in phase space calls for further investigations.

Multi-strange baryons $\left(\Xi^{-}, \Omega\right)$ are expected to be a sensitive probe of baryon-rich QCD matter [61]. In fact, HADES provided quite intriguing pioneering results on double strange $\Xi^{-}$hyperons. For the first time $\Xi^{-}$have been reconstructed at such low collision energies $[62,63]$ (see left and middle panels of Fig. 6). It should be emphasized that the $\Xi^{-}$hyperon is measured $640 \mathrm{MeV}$ below the respective $\mathrm{N}+\mathrm{N}$ threshold in $\mathrm{Ar}+\mathrm{KCl}$ collisions at $1.76 \mathrm{~A} \mathrm{GeV}$. Fig. 6 (right panel) shows the ratio of production rate of $\Xi^{-}(1321)$ and $\Lambda+\Sigma^{0}$ as a function of the total center-of-mass energy in $\mathrm{N}+\mathrm{N}$ collisions [63]. The observed multiplicity of $\Xi^{-}$is by a factor $15 \pm 6$ higher than the prediction based on SHM, commonly considered to be an upper limit for particle production in heavy-ion collisions. Also transport approach underestimates the experimental ratio (for both 

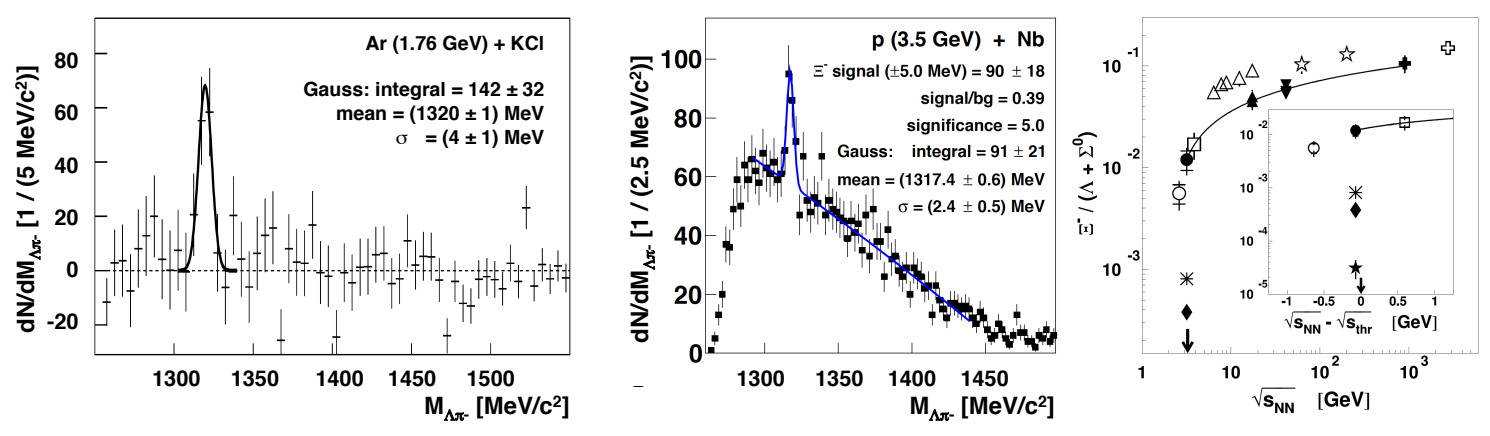

Figure 6: Left: The experimental $\Lambda \pi^{-}$invariant mass distribution reconstructed in $\mathrm{Ar}+\mathrm{KCl}$ collisions at $1.76 \mathrm{~A} \mathrm{GeV}$. Error bars show the statistical errors. The solid line represents a Gaussian fit to $\Xi^{-}$signal after background subtraction. Middle: Same in $\mathrm{p}+\mathrm{Nb}$ collisions at $3.5 \mathrm{GeV}$. The curve represents a combination of a Gaussian and a polynomial function used to fit the data. Right: The yield ratio $\Xi^{-} /\left(\Lambda+\Sigma^{0}\right)$ as a function of $\sqrt{s_{N N}}$ or $\sqrt{s_{N N}}-\sqrt{s_{t h r}}$ (insert). The arrows indicate the threshold in free $\mathrm{N}+\mathrm{N}$ collisions.

$\mathrm{Ar}+\mathrm{KCl}$ and $\mathrm{p}+\mathrm{Nb}$ systems) and it is necessary to better understand e.g. the strangeness-exchange reactions conjectured as the dominant process for cascade production below and close to threshold $[66,67]$. A possible explanation of this observation, suggesting production from high mass $\left(>2 \mathrm{GeV} / c^{2}\right)$ baryonic resonances with significant branching ratios for the $\Xi^{-}$decay, has been proposed in [64]. However, to finally conclude on existence of such decays dedicated measurements with $\mathrm{p}+\mathrm{p}$ or $\pi^{-}+\mathrm{p}$ experiments are required. It is important to notice that model is instrumental in describing the yields of $\phi$ and $\Xi^{-}$only if "secondary" reactions are taking place, leading to excitation of various baryonic resonances. Furthermore, the corresponding particle yields can be well described with the thermal model and resemble yields of a hadronic system in chemical equilibrium. In fact only $2-3$ inelastic collisions sufficient to create a system which appears to be in chemical equilibrium [54, 68]. The strange hadrons $\mathrm{K}^{-}, \mathrm{K}_{s}^{0}, \Lambda$ and $\phi$ have been reconstructed for the first time in $\mathrm{Au}+\mathrm{Au}$ collisions at even lower collision energy of $1.23 \mathrm{~A} \mathrm{GeV}$. These measurements will put strong constraints on production and propagation mechanisms of strangeness in strongly interacting matter.

\section{Exploring the nuclear equation of state}

The knowledge of the nuclear EoS at supra-normal densities is essential for our understanding of the nuclear forces as well as for astrophysical purposes. Several conclusions made in the past $[69,70]$ need to be revised. Specifically, the extracted repulsive $\mathrm{K}^{+}$-nucleon potential and the corresponding soft nuclear EoS suggested that the maximum gravitational mass of a compact (neutron) star cannot be larger than two solar masses. The onset of hyperons in the core of neutron stars and the consequent softening of the equation of state have also been questioned for a long time. The recent observations of the pulsars PSR J1614-2230 and PSR J0348+0432 proves the existence of $2 \mathrm{M}_{\odot}$ neutron stars [71]. Many models with hyperons fail to describe a $2 \mathrm{M}_{\odot}$ pulsar mass [72]. Does the breakdown of baryonic models at high densities signal onset of a new phase with non-baryonic degrees of freedom? Two scenarios were proposed to solve the so called 

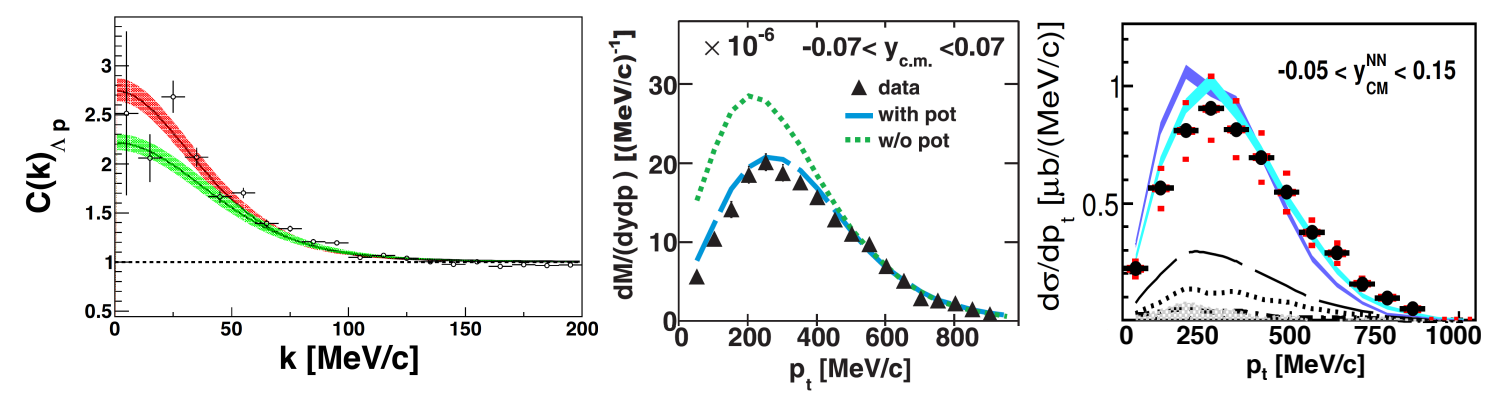

Figure 7: Left: Transverse momentum ( $p_{t}$ distribution of the $\mathrm{K}_{s}^{0}$ reconstructed in $\mathrm{Ar}+\mathrm{KCl}$ collisions at $1.76 \mathrm{~A} \mathrm{GeV}$ (full triangles) together with the results of the $\mathrm{QQMD}$ model including a repulsive $\mathrm{K}^{0}$-nucleus potential of $46 \mathrm{MeV}$ (dashed curves) and without potential (dotted curves). Middle: Same in p+Nb collisions at $3.5 \mathrm{GeV}$, cyan curve - with, violet curve - w/o potential. Right: Comparison of the experimental $\Lambda p$ correlation function (open circles with error bars) to the LO (green) and NLO (red) scattering parameter set (see for details [75]). The error bands in the theory curves correspond to the errors of the $\Lambda \mathrm{p}$ source size determination.

"hyperon puzzle": (i) additional repulsion between hyperons due to a vector meson coupled to hyperons only; (ii) a transition to a very stiff quark plasma before the hyperon threshold [73].

More precise data is needed to clarify to what extent these differences indicate residual systematic uncertainties or reflect the general statistical limits of the presently available experimental data. Various observables have been suggested to address this question [74]. Our programme of investigations of the in-medium kaon potential has been started with the measurement down to low transverse momentum of $\mathrm{K}_{s}^{0}$ production in $\mathrm{Ar}+\mathrm{KCl}$ [76] and in $\mathrm{p}+\mathrm{Nb}$ [77] collisions revealing a strong repulsive vector potential of about $40 \mathrm{MeV}$. New HADES Au+Au data will allow to constrain models further.

To contribute to the question of hyperon-nucleon and hyperon-nucleon-nucleon interaction we use the femtoscopy technique to study the scattering lengths and effective ranges for hyperonnucleon pairs produced in $\mathrm{p}+\mathrm{Nb}$ collisions [78] (an alternative to scattering experiments). The final state interaction strength between $\Lambda \mathrm{p}$ was investigated by comparing the experimental $\Lambda \mathrm{p}$ correlation function to model calculations using scattering parameters from chiral effective field theory computations [75]. A comparison of the correlation functions using the leading-order (LO, green band) and next-to-leading-order (NLO, red band) scattering parameters are shown in Fig. 7 (right panel). The statistics was not enough to clearly distinguish between model predictions, but increase in statistics by a good order of magnetite is expected with upcoming p+A program.

\section{Résumé and prospects}

HADES provides high-quality data for developping a thorough understanding of the dielectron and strangeness production in elementary and heavy-ion collisions at the SIS energy range. Our recent results provided the encouraging prospects for studying QCD matter in the region of nonzero net-baryon density. Our observations on electromagnetic radiation and rare (multi-)strange hadron production are in accordance with a picture describing the fireball as strongly interacting resonance gas. 
Further experiments on baryonic-resonance and baryon-rich QCD matter will be realized at SIS18 during FAIR Phase-0. Actually, HADES is at the moment the only facility world-wide which combines a pion beam with dilepton spectrometry and acts as a precursor in view of existing plans for meson beam facilities for baryon spectroscopy. Emphasis will be placed on the electromagnetic transition form factors of baryonic resonances, the coupling of vector mesons and kaons to baryons and medium effects in "cold" nuclear matter. High statistics $\mathrm{p}+\mathrm{p}$ and $\mathrm{p}+\mathrm{A}$ runs with proton beam energies of $4.5 \mathrm{GeV}$ (maximum SIS18 energy) would enable very important studies of $\omega$ and $\phi$ production and propagation, in both the hadronic and the leptonic final states, strange meson and multi-strange baryon production, excited hyperon states and the first measurements of their electromagnetic Dalitz decays.

The combined measurement of dielectrons and strangeness in $\mathrm{Ar}+\mathrm{KCl}$ and $\mathrm{Au}+\mathrm{Au}$ collisions has provided intriguing results which call for further systematic investigations. We propose an experiment which focuses on measuring a medium-heavy collision system at the maximum energy of SIS18, to increase the NN center-of-mass energy in favour of an enhanced strangeness production. Silver (stripped to charge state $45^{+}$) could be accelerated in SIS18 to $1.67 \mathrm{~A} \mathrm{GeV}$. Particular emphasis will be put on the systematic investigation of the multi-differential emission probability of dileptons in the intermediate-mass range $\left(>1 \mathrm{GeV} / c^{2}\right)$. These measurements will provide unique insight into the microscopic properties of matter under extreme conditions and can signal onset of chiral symmetry restoration $[79,80]$.

\section{References}

[1] A. Andronic et al., Nucl. Phys. A 837 (2010) 65;J. Stachel et al., J.Phys.Conf.Ser. 509 (2014) 012019

[2] F. Becattini et al., Phys. Lett. B 764 (2017) 241

[3] J. Cleymans et al., Phys. Rev. C 73 (2006) 034905

[4] L. Adamczyk et al. [STAR Collaboration], arXiv:1701.07065 [nucl-ex].

[5] G. Agakishiev et al. [HADES Collaboration], Eur. Phys. J. A 52 (2016) no. 6, 178

[6] X. Lopez et al. [FOPI Collaboration], Phys. Rev. C 76 (2007) 052203

[7] O. Kaczmarek et al., Phys. Rev. D 83 (2011) 014504

[8] S. Borsanyi et al. [Wuppertal-Budapest Collaboration], JHEP 1009 (2010) 073

[9] H. J. Specht et al. [NA60 Collaboration], AIP Conf. Proc. 1322 (2010) 1

[10] Z. Fodor and S. D. Katz, JHEP 0404 (2004) 050

[11] B. J. Schaefer, J. M. Pawlowski and J. Wambach, Phys. Rev. D 76 (2007) 074023

[12] L. McLerran and R.D. Pisarski, Nucl. Phys. A 796 (2007) 83

[13] L. McLerran, Ch. Sasaki and K. Redlich, Nucl. Phys. A 824 (2009) 86

[14] A. Andronic et al., Nucl. Phys. A 837 (2010) 65

[15] K. Fukushima and C. Sasaki, Prog. Part. Nucl. Phys. 72 (2013) 99

[16] J. Steinheimer and J. Randrup, Phys. Rev. Lett. 109 (2012) 212301

[17] S. Carignano and M. Buballa, Phys. Polon. Supp. 5 (2012) 641

[18] A. Kurkela and A. Vuorinen, Phys. Rev. Lett. 117, no. 4 (2016) 042501

[19] B. Friman et al., Lect. Notes Phys. 814 (2011) pp.1.

[20] C. Fuchs, Prog. Part. Nucl. Phys. 56 (2006) 1-103

[21] C. Hartnack et al., Phys. Rept. 510 (2012) 119

[22] G. Agakishiev et al. [HADES Collaboration], Eur. Phys. J. A 41 (2009) 243

[23] T. Ablyazimov et al. [CBM Collaboration], Eur. Phys. J. A 53, no. 3 (2017) 60

[24] J-PARC-HI proposal http://silver.j-parc.jp/sako/white-paper-v1.21.pdf

[25] A. Dainese et al., Frascati Phys. Ser. 62 (2016)

[26] R. Rapp and H. van Hees, Phys. Lett. B 753 (2016) 586

[27] A. Adare et al. [PHENIX Collaboration], Phys. Rev. C 93 (2016) no.1, 014904 
[28] L. Adamczyk et al. [STAR Collaboration], Phys. Rev. C 92 (2015) no.2, 024912

[29] L. Adamczyk et al. [STAR Collaboration], Phys. Lett. B 750 (2015) 64

[30] R. Rapp and J. Wambach, Adv. Nucl. Phys. 25, 1 (2000)

[31] M. Herrmann, B. L. Friman and W. Norenberg, Nucl. Phys. A 560, 411 (1993)

[32] G. Chanfray and P. Schuck, Nucl. Phys. A 555, 329 (1993)

[33] R. Rapp, G. Chanfray and J. Wambach, Phys. Rev. Lett. 76 (1996) 368

[34] W. Peters, M. Post, H. Lenske, S. Leupold and U. Mosel, Nucl. Phys. A 632, 109 (1998)

[35] G. Agakishiev et al. [HADES Collaboration], Eur. Phys. J. A 48 (2012) 64

[36] J. Weil, H. van Hees and U. Mosel, Eur. Phys. J. A 48 (2012) 111

[37] L. P. Kaptari and B. Kampfer, Phys. Rev. C 80 (2009) 064003

[38] R. Shyam and U. Mosel, Phys. Rev. C 79 (2009) 035203

[39] R. Shyam and U. Mosel, Phys. Rev. C 82 (2010) 062201

[40] G. Ramalho, M. T. Pena, J. Weil, H. van Hees and U. Mosel, Phys. Rev. D 93 (2016) no.3, 033004

[41] F. Scozzi [HADES Collaboration], EPJ Web Conf. 130 (2016) 07021

[42] W. Przygoda [HADES Collaboration], JPS Conf. Proc. 10 (2016) 010013

[43] M. Post, S. Leupold and U. Mosel, Nucl. Phys. A 741 (2004) 81

[44] R. Rapp, AIP Conf. Proc. 1322 (2010) 55

[45] M. Naruki et al., Phys. Rev. Lett. 96 (2006) 092301

[46] R. Nasseripour et al. [CLAS Collaboration], Phys. Rev. Lett. 99 (2007) 262302

[47] G. Agakishiev et al. [HADES Collaboration], Phys. Lett. B 715 (2012) 304

[48] G. Agakishiev et al. [HADES Collaboration], Eur. Phys. J. A 50 (2014) 82

[49] R. Arnaldi et al. [NA60 Collaboration], Phys. Rev. Lett. 96 (2006) 162302

[50] G. Agakishiev et al. [HADES Collaboration], Phys. Lett. B 690 (2010)118

[51] G. Agakishiev et al. [HADES Collaboration], Phys. Rev. C 84 (2011) 014902

[52] P. Tlusty et al. [HADES Collaboration], arXiv:0906.2309 [nucl-ex]

[53] R. Averbeck et al. [TAPS Collaboration], Z. Phys. A 359 (1997) 65

[54] T. Galatyuk, P. M. Hohler, R. Rapp, F. Seck and J. Stroth, Eur. Phys. J. A 52, no. 5 (2016) 131

[55] S. Endres, H. van Hees, J. Weil and M. Bleicher, Phys. Rev. C 92, no. 1 (2015) 014911

[56] E. L. Bratkovskaya et al., Phys. Rev. C 87 (2013) 064907

[57] J. Weil, J. Staudenmaier and H. Petersen, J. Phys. Conf. Ser. 742 (2016) no.1, 012034

[58] G. Agakishiev et al. [HADES Collaboration], Phys. Rev. C 80 (2009) 025209

[59] S. Wheaton and J. Cleymans, Comput. Phys. Commun. 180 (2009) 84

[60] P. Koch, B. Muller and J. Rafelski, Phys. Rept. 142 (1986) 167.

[61] K. Fukushima, Eur. Phys. J. A 52 (2016) no.8, 222.

[62] G. Agakishiev et al. [HADES Collaboration], Phys. Rev. Lett. 103 (2009)132301

[63] G. Agakishiev et al. [HADES Collaboration], Phys. Rev. Lett. 114 (2015) no.21, 212301

[64] J. Steinheimer and M. Bleicher, J. Phys. G 43 (2016) no.1, 015104

[65] H. Schade, G. Wolf and B. Kampfer, Phys. Rev. C 81 (2010) 034902

[66] F. Li, L. W. Chen, C. M. Ko and S. H. Lee, Phys. Rev. C 85 (2012) 064902

[67] E. E. Kolomeitsev, B. Tomasik and D. N. Voskresensky, Phys. Rev. C 86 (2012) 054909

[68] J. Steinheimer et al., Phys. Rev. C 93 (2016) no.6, 064908

[69] C. Fuchs, A. Faessler, E. Zabrodin, and Y.-M. Zheng, Phys. Rev. Lett. 86 (2001) 1974

[70] C. Hartnack, H. Oeschler, and J. Aichelin Phys. Rev. Lett. 96 (2006) 012302

[71] P. Demorest et al., Nature 467 (2010) 1081; J. Antoniadis et al., Science 340 (2013) 6131

[72] D. Lonardoni, A. Lovato, S. Gandolfi and F. Pederiva, Phys. Rev. Lett. 114 (2015) no.9, 092301

[73] M. Buballa et al., J. Phys. G 41 (2014) no.12, 123001

[74] P. Russotto et al., Phys. Rev. C 94 (2016) no.3, 034608; Z. G. Xiao et al., Eur. Phys. J. A 50 (2014) 37

[75] J. Haidenbauer et al., Nucl. Phys. A 915 (2013) 24

[76] G. Agakishiev et al. [HADES Collaboration], Phys. Rev. C 82 (2010) 044907

[77] G. Agakishiev et al. [HADES Collaboration], Phys. Rev. C 90 (2014) 054906

[78] J. Adamczewski-Musch et al. [HADES Collaboration], Phys. Rev. C 94, no. 2 (2016) 025201

[79] P. M. Hohler and R. Rapp, Phys. Lett. B 731 (2014) 103; Phys. Rev. D 89 (2014) no.12, 125013

[80] C. Jung et al., Phys. Rev. D 95 (2017) no.3, 036020 